\title{
Chinese Herbal Preparation SaiLuoTong Alleviates Brain Ischemia Via Nrf2 Antioxidation Pathway Dependent Cerebral Microvascular Protection
}

\section{Xiao-Di Fan ( $\square$ xiaodi.1018@163.com )}

China Academy of Chinese Medical Sciences Xiyuan Hospital https://orcid.org/0000-0002-5004-7099

\section{Ming-Jiang Yao}

China Academy of Chinese Medical Sciences

\section{Bin Yang}

China Academy of Chinese Medical Sciences Xiyuan Hospital

\section{Xiao Han}

China Academy of Chinese Medical Sciences Xiyuan Hospital

\section{Guang-Rui Wang}

China Academy of Chinese Medical Sciences Xiyuan Hospital

\section{Peng Li}

China Academy of Chinese Medical Sciences Xiyuan Hospital

\section{Li Xu}

China Academy of Chinese Medical Sciences Xiyuan Hospital

\section{Research}

Keywords: cerebral ischemia, SaiLuoTong capsule, brain microvascular endothelial cells, anti-oxidation, nuclear factor-E2-related factor 2, siRNA interference

Posted Date: January 7th, 2021

DOI: https://doi.org/10.21203/rs.3.rs-139872/v1

License: (c) (1) This work is licensed under a Creative Commons Attribution 4.0 International License. Read Full License 


\section{Abstract}

Background: Stroke is one of the most devastating diseases worldwide. Chinese herbal preparation SaiLuoTong capsule (SLT) showed outstanding therapeutic effects over stroke and its sequelae. This study is to elucidate the mechanism.

Methods: We duplicated cerebral ischemia model in rats by permanent MCAO, and simulated ischemia injury in cultured hCMEC/D3 cells with oxygen and glucose deprivation/reoxia (OGD/R); we treated them with SLT. Brain infarction volumes and micromorphology were examined with TTC and HE stainings, respectively; neurological function injuries were valued with deficits scoring; brain water contents were measured with the wet-dry method; and expressions of claudin-1, occludin, Nrf2 and HO-1 were examined with western blot assay and immunohistochemstry or immunocytofluorescnce stainings; activities of SOD and contents of GSH were measured by colorimetric method; cell viabilities were measured by CCK8 method; cell monolayer permeabilities were assessed by FITC-dextran diffusion method.

Results: SLT (33 mg/kg) significantly decreased infarction volumes, relieved neuron degenerations and neuron loss, and ameliorated neurological functions; SLT also significantly inhibited elevations in brain water content and decreases of claudin-1 and occludin expressions; additionally SLT significantly increased the nucleus translocation of Nrf2, elevated expression of HO-1, and raised activity of SOD and content of GSH ( $p<0.05$ or 0.01 , compared with the model group, in quantitative data). These testified SLT's anti-stroke effect, and hint the possible key role of cerebral blood vascular endothelial cells (CBVEC) protection and Nrf2 pathway in SLT's therapy. In hCMEC/D3 cells, SLT significantly inhibited the drop in cell viabilities; SLT also significantly facilitated the nucleus translocation of Nrf2, and increased the expression of HO-1, the activity of SOD, and the content of GSH (in comparison to the model group, $\mathrm{p}<0.05$ or 0.01 ). Lastly, the anti-OGD/R effects of SLT in hCMEC/D3 cells, including raising cell viabilities, inhibiting the elevation in cell monolayer permeabilities, and preserving the expressions of claudin- 1 and occludin, were all abolished by Nrf2 siRNA interference. These undoubtedly confirmed SLT's protective effect on CBVEC and the obligatory role of Nrf2 pathway in it.

Conclusion: SLT's therapeutic effect on brain ischemia is related to its protection on CBVEC, which is dependent on the activation of Nrf2 pathway.

\section{Background}

Stroke is one of the leading causes of morbidity and mortality worldwide, which produces immense healthy and economic burdens. Taking the case of China, persons of tens of millions suffer stroke each year, nearly ten percent of them die, and most of the rest are afflicted in different extents by the sequelae such as sensory and motor impediments, cognition impairments, and affective and speech disorders, which badly influences their lives as well as their families'.

Most of stroke are ischemic type, which occupies $80 \%$ of the total cases. It is usually triggered by obstructions of one or more cerebral arteries, which then lead to a critical reduction of regional cerebral 
blood flow, causing a waterfall-like cascade, and finally resulting in massive neuron deaths [1]. In this damage cascade, the ruin of brain blood barrier (BBB) plays a pivotal role.

The endothelium of cerebral blood microvessels is unique to that in other tissues, as they have continuous intercellular tight junctions (TJs) and efflux transporters, thus the endothelium and their TJs form a barrier-like structure, which can greatly limit both the paracellular and transcellular diffusion of vascular inclusions, and constitute BBB [2,3].

Growing evidences demonstrate that brain ischemia causes loss of endothelial cells and TJs, leading to enhanced BBB permeability not only including extravasations of blood plasma constituents and some neurotoxins, but also including the infiltrations of neutrophils and monocytes, which further causes neuron damages, significantly amplifying the effects of ischemia and making the injury irreversible $[4,5,6]$.

Oxidative stress is the major cause of BBB damages in ischemia, which refers to a state in that the generation of reactive oxygen species (ROS) exceeds far behind the body's dispose ability, leading to serious impairments $[7,8]$. Redressing this imbalance between ROS and ROS scavenging in the brain blood vascular endothelial cells is necessary and urgent for the stroke treatment. In comparison to direct eliminating ROS, inspiring the innate antioxidation system is a better choice for it has a higher efficiency, longer effecting duration and larger safety $[7,8]$.

The nuclear factor erythroid 2-related factor 2 (Nrf2) belongs to Cap ' $n$ ' Collar (Cnc)-bZIP (basic leucine zipper) family, and is a transcription factors regulating the expressions of a series of anti-oxidant, antiinflammatory and detoxifying proteins [9]. Nrf2 pathway is the most important antioxidation machinery of the body and is also closely related to cerebral blood vascular endothelium preservation and the outcome of brain ischemia.

A large number of studies showed that the activation of Nrf2 pathway can lead to strong anti-oxidation and anti-apoptosis effects and BBB protection in the brain infarction [10-16]. In contrast, mass reports exhibited as well that the deletion or downregulation of Nrf2 exacerbated brain injuries in ischemia, in which the acceleratedly destroyed TJs in cerebral blood vessels and increased BBB breakdown and brain edema played a pivotal role $[11,17]$. These results indicate that Nrf2 pathway is a pivotal target for vascular endothelial cells protection and stroke therapy.

SaiLuoTong capsule (SLT) is an outstanding representative of new type Chinese herbal preparation, which is composed with refined herbal extracts, instead of crude drugs, thus having a definite and controllable composition, in which the high efficiency ingredients are enriched, and the lower and even inefficiency ones are removed. This characteristic renders SLT to overcome the shortcomings of traditional Chinese herbal preparations, significantly enhances the controllability in production and safety, and increases the therapeutic effects. 
SLT is composed of extracts of three Chinese herbal medicines, i.e. the roots of Panax ginseng (ginseng), the leaves of Ginkgo biloba (ginkgo), and the flowers of Crocus sativus (saffron) with the proportion of 5:5:1 that is derived from pharmacodynamical optimization experiments in animals [1819]. The main active components of SLT are ten compounds including three ginsenosides, three flavones, three ginkgolides, and one crocin (the details of them are listed in table 1).

Numerous studies have testified the remarkable protective effect of SLT against brain ischemia. And the therapeutic mechanism was revealed as anti-inflammation, anti-oxidative stress, and anti-apoptosis and platelet aggregation, as well as improving blood flow and brain tissue acetylcholine (ACh) content [19-23]. More encouragingly, in a strictly designed clinical trial with multiple centers, large sample, and double blinded placebo control, SLT showed a significant ameliorative effect in patients with mild to moderate vascular dementia, meanwhile no toxicities were exhibited [18-19]. These protruding achievements suggest that SLT may have a glorious prospect in the treatments of stroke and its sequelae, thus the exploration on its therapeutic mechanisms should be an important and long lasting issue.

The relationship between SLT's anti-stroke effect and Nrf2 pathway mediated cerebral vascular endothelial cells protection has not been clarified. In the present study, we investigated this issue with experiments in both rats and cultured cells. This study may be significant for clarifying the therapeutic mechanism of SLT on stroke, and for searching effective drugs for stroke remedy.

\section{Methods}

\section{Animal preparation}

Male Sprague-Dawley rats (200-230g) were purchased from SPF (Beijing) Biotechnology Co., Ltd. (Beijing, China, No.1103241911033018). Rats were maintained in an air-conditioned room (temperature: $21 \pm 2^{\circ} \mathrm{C}$ ) under a $12 \mathrm{~h}$ day-night cycle with free access to food and water, and were acclimated for 3 days prior to the experiment. Animal handling procedures were performed in accordance with the guide of the Ethics Committee of Xi Yuan Hospital of China Academy of Chinese Medical Sciences (Protocol No. 2019XLC015-2). And all animal housing, care, feeding, and experimental procedures were in compliance with the National Guidelines for Animal Protection.

\section{Establishment of cerebral infarction model in rats with permanent middle cerebral artery occlusion (pMCAO)}

Rats were anesthetized by an intraperitoneal injection with $1 \%$ pentobarbital sodium $(80 \mathrm{mg} / \mathrm{kg})$. Under anesthesia, the right common carotid, the right external carotid, and the right internal carotid were separated and were carefully exposed. The right external carotid and the right common carotid were ligated with a suture silk. Thereafter, a 3-0 monofilament nylon suture with a rounded tip diameter $0.32 \mathrm{~mm}$ (Item\#2432A1, Beijing Sunbio Biotech Co Ltd) was introduced into the bifurcation of the right 
common carotid and then was intracranially advanced for approximately $18 \mathrm{~mm}$ to block the blood flow of the right middle cerebral artery. During this procedure, the body temperature was maintained at $37^{\circ} \mathrm{C}$ with a warm pad. For the sham-operated group, only skin incisions were performed under anesthesia.

\section{Drug treatments in rats}

SLT was provided by the ShenWei Pharmaceutical Corporation (Heibei, China). SLT was soluted in saline, and was injected into the duodenum immediately after the right middle cerebral artery was blocked. The doses of SLT were set as $16.5 \mathrm{mg} / \mathrm{kg}$ (SLT-L) and $33 \mathrm{mg} / \mathrm{kg}$ (SLT-H). Rats in the sham group and model group were injected with saline in the same way and at the same time point.

\section{Measurement of neurological deficits}

The neurological function deficit scores of rats were blindly evaluated 24h after MCAO. A 5 point scale was used as follows: 0 , no neurological deficits; 1 , failure to fully extend the left forelimb; 2 , decreased resistance to a lateral push toward the right side and failure to fully extend the left forepaw; 3 , circling to the left side; and 4, inability to walk spontaneously and lack of response to stimulation [24].

\section{Assessment of infarct volume}

After neurological function tests, the rats were killed by decapitation, and their brains were taken out and were sectioned into slices of $2 \mathrm{~mm}$ thickness; the slices were incubated in a 2\% 2,3,5-Triphenyltetrazolium chloride (TTC) solution at $37^{\circ} \mathrm{C}$ for 15 minutes. TTC stained the noninfarcted region with a deep red pigment, while the infarcted brain areas were stained with white [24]. Stained sections were photographed, and the images were analyzed to calculate the infarct volumes by using an Image ProPlus 6.0 analysis system (Media Cybernetics, Rockville, MD, USA).

\section{Brain tissue fixations, embeddings, and sections and HE stainings}

$24 \mathrm{~h}$ after $\mathrm{MCAO}$, the rats were killed by decapitation, and the brains were then rapidly taken out and were fixed in $4 \%$ paraformaldehyde for 7 days. The brains were then embedded in paraffin and were sectioned into slices of $7 \mu \mathrm{m}$. The sections were stained with HE and were observed with an Olympus BX51 microscope.

\section{Evaluation of blood water content of the brain}

24h after MCAO, rats were sacrificed by decapitation, and their brains were taken out. The wet brains were weighed and were dried at $60^{\circ} \mathrm{C}$ for 3 days, and then the weights of the dry brains were measured. The brain water content $=(1-$ dry weight $/$ wet weight $) \times 100 \%$.

\section{Immunohistochemistry examinations}

The brain sections were deparaffinized, rehydrated, and blocked. Next, the sections were incubated overnight at $4^{\circ} \mathrm{C}$ with anti-claudin- 1 antibody (1:500, ab15098, abcam), anti-occludin antibody (1:500, 
27260-1-AP, proteintech), and anti-heme oxygenase-1 (HO-1) antibody (1:500, 66743-1-Ig, proteintech), respectively. The sections were rinsed with PBS for three times, then were incubated with horseradish peroxidase (HRP)-conjugated goat anti-rabbit $\lg$ at $37^{\circ} \mathrm{C}$ for $20 \mathrm{~min}$, and were colorized with DAB. Lastly, hematoxylin restaining was performed. The sections were observed under an Olympus BX51 microscope.

\section{Western blot assay}

The nuclear and cytosol protein extractions for Nrf2 and total protein extractions for Nrf2, HO-1, occludin, and claudin 1 from the samples (in in vivo experiments, were cerebral cortex; in in vitro experiment, were hCMEC/D3 cells) were performed by using the nuclear-cytosol extraction kit (Applygen Technologies Inc., Beijing) or the total protein extraction kit (Solarbio Science $₫$ Technology Co. Ltd Beijing). Equal amounts of protein $(50 \mu \mathrm{g})$ were loaded into $10 \%$ or $12.5 \%$ SDS-PAGE gels, and then were subjected to electrophoresis, lastly were transferred to nitrocellulose membranes (Millipore, Billerica, MA, USA). The membranes were blocked with $5 \%$ bovine serum albumin (BSA) for $1 \mathrm{~h}$ at room temperature, and then were incubated overnight at $4{ }^{\circ} \mathrm{C}$ with anti-claudin-1 antibody (1:1000), anti-occludin antibody (1:1000), anti-Nrf2 antibody (1:1000), anti-HO-1 antibody (1:1000), anti-GAPDH antibody (1:1000,60004-1-lg, proteintech), anti- $\beta$-actin antibody $(1: 1000,60008-1-\mathrm{Ig}$, proteintech) or anti-histone $\mathrm{H} 3$ antibody (17168-1AP, proteintech). The membranes were incubated with HRP-conjugated secondary antibodies for $1.5 \mathrm{~h}$ at room temperature. The protein bands were enlightened with an enhanced chemiluminescence kit, and their brightness were quantified by using Image LabTM Software.

\section{Determination of the activities of superoxide dismutase (SOD) and the contents of glutathione (GSH)}

All samples (brains and cells) were made into homogenates by an ultrasounic cell disrupter at $0{ }^{\circ} \mathrm{C}$. SOD activities and GSH contents in the homogenates were analyzed with the merchant kits (Institute of Biological Engineering of Nanjing Jiancheng, Nanjing, China).

\section{Cell culture}

Human brain microvascular endothelial cell line (hCMEC/D3, iCellBioscience, Inc. Shanghai, China) were cultured at $37^{\circ} \mathrm{C}$ with $5 \% \mathrm{CO}_{2}$ in Endothelial Cell Medium (ECM, PriMed-iCell-0016, China) supplemented with $5 \%$ fetal bovine serum, $1 \%$ ECGS, $100 \mathrm{U} \cdot \mathrm{mL}^{-1}$ penicillin and $100 \mu \mathrm{g} \cdot \mathrm{mL}^{-1}$ streptomycin, and were passaged with $0.25 \%$ trypsin.

\section{Oxygen-glucose deprivation and reoxygenation (OGD/R) model and SLT treatments}

For oxygen-glucose deprivation (OGD), hCMEC/D3 cells were incubated in a glucose-free Dulbecco's Modified Eagle's Medium (DMEM, Gibco, USA) in a customer-made chamber filled with $95 \% \mathrm{~N}_{2} / 5 \%$ $\mathrm{CO}_{2}$ for $4 \mathrm{~h}$ at $37^{\circ} \mathrm{C}$. After OGD, for reoxygenation, the incubation media was replaced with normal ECM and the cells were cultured under normal atmosphere with $5 \% \mathrm{CO}_{2}$. 
The mock group was incubated in normal DMEM with $5 \% \mathrm{CO}_{2}$ for $4 \mathrm{~h}$ at $37^{\circ} \mathrm{C}$, and was then incubated with ECM.

SLT was soluted with DMSO, and the solutions were added into the incubation medium with the volume ratio of 1:1000 at the beginnings of OGD and reoxygenation. The mock group and the OGD/R model group were treated with DMSO in the same way at the same time points.

\section{Cell viability}

The cells were incubated with CCK8-ECM solution $(1: 10)$ at $37^{\circ} \mathrm{C}$ for $2 \mathrm{~h}$. The absorbance at $450 \mathrm{~nm}$ was measured with a microplate reader. Then the cell viabilities were obtained through normalization to the average absorbance of the mock (normal control) group.

\section{Immunofluorescence assay}

Paraformaldehyde-fixed hCMEC/D3 cells were incubated with anti-Nrf2 antibody or anti-HO-1 antibody (both 1:200), followed by the incubation with secondary antibodies, i.e. Fluorescein-Conjugated AffiniPure Goat anti-Rabbit IgG (1:100, Yuabio, china), and were stained with DAPI finally. Cells were observed and imaged with an Olympus IX81 live cell station.

\section{RNA interference by siNrf2 and drug treatment}

Transfections in HCMEC/D3 cells were conducted with Lipofectamine 3000 reagent (Thermo Fisher Scientific, USA). High purity control siRNAs (negative control siRNA and GAPDH siRNA) and Nrf2 siRNAs were obtained from JTSBIO (Wuhan, China). The Nrf2 siRNA sequences were: Nrf2 siRNA-1, forward, CCCUGAAAGCACAGCAGAATT, and reverse, UUCUGCUGUGCUUUCAG- GGTT; Nrf2 siRNA-2, forward, CCAGAACACUCAGUGGAAUTT, and reverse, AUUCCACUGAGUGUUCUGGTT; Nrf2 siRNA-3, forward, GCCUGUAAGU- CCUGGUCAUTT, and reverse, AUGACCAGGACUUACAGGCTT. Negative control (NC) siRNA sequences were: forward, UUCUCCGAACGUGUCACGUTT, and reverse, ACGUGACACGUUCGGAGAATT. GAPDH sequences were: forward, UGACCUCAACUACAUGGUUTT, and reverse, AACCAUGUAGUUGAGGUCATT.

\section{Paracellular Permeability Measurement}

Cell monolayer integrity was assessed by diffusion of fluorescein isothiocyanate (FITC)-dextran (4kDa, Lot:64878, MCE, USA) as previously described [13]. After OGD/R, $400 \mu \mathrm{L}$ FITC-dextran $(0.5 \mathrm{mg} / \mathrm{mL})$ solutions were added to the upper chamber of 12-well transwell culture plate inserts (A190059, Millicell, Germany), on the bottom of which the hCMEC/D3 cell confluents grew. Inserts were placed in 12-well culture plates containing $1000 \mu \mathrm{L}$ of DMEM/F12 media (serum-free and without phenol red) per well. Then the cells were incubated at $37^{\circ} \mathrm{C}$ for $60 \mathrm{~min}$ in the dark. Inserts were removed and the solutions in the wells were collected and transferred into a back-96-well plate. The fluorescence intensities were measured by using the excitation and emission wavelengths as 490 and $520 \mathrm{~nm}$, respectively, and were converted to the concentrations of FITC-dextran with the calibration curve. 


\section{Statistical analysis}

The data are expressed as Mean \pm SEM and were statistically analyzed by using the Statistical Product and Service Solutions (SPSS) 16.0 software; data comparisons between two groups were conducted with t-test, that among multiple groups were conducted with one-way or two-way analysis of variance (ANOVA). The statistical significance level was set to 0.05 , i.e. differences were deemed statistically significant as $\mathrm{P}<0.05$.

\section{Results}

\section{SLT attenuated cerebral infarctions and improved the neurological functions in MCAO rats}

No infarction was detected in the sham group, while in the model group, infarctions of large volumes were developed. SLT treatments alleviated the brain infarctions, and the infarct volumes in SLT-H group were significantly lower than that in the model group $(P<0.05$, Fig. $1 a, b)$. Accordingly, in the brain microscopy, no neuron damage, neuron loss or other notable morphological abnormalities were shown in the sham group; however, necroses of large areas were observed in the model group, in which severe neuron loss, and neuron cell degenerations including cytoplasm acidophilic degeneration, nuclear pyknosis were shown; in the regions peripheral to necrosis, tissue edema and neuron hydropic degeneration were shown; and these lesions were notably attenuated in SLT treated groups, especially in SLT-H group (Fig.1c).

Neurological deficit scores were significantly elevated in the model group in comparison to the sham group $(P<0.01)$, indicating a severe injury in neurological function was induced by brain ischemia. However, treatments with SLT alleviated the elevation in neurological deficit scores, especially with the high dose, of which the effect was significant $(P<0.05$, compared with the model group; Fig. $1 d)$

\section{SLT decreased water content of the brain and inhibited the drop in expressions of claudin- 1 and occludin in MCAO rats, implying cerebral microvascular protection may underlie its anti-brain ischemia effect}

The water contents of the infarcted hemispheres in the model group were significantly increased in comparison to the ipsilateral hemispheres in the sham group $(P<0.01)$; however, that in SLT groups were decreased, especially in the SLT-H group, which were significantly lower than that in the model group $(P<0.01$, Fig.2a). These results hint that SLT may have the effect of protecting cerebral blood microvessels, thus alleviating its leakage.

Additionally, the examinations on claudin-1 and occludin, two key TJs-related proteins that play pivotal roles in maintaining BBB integrity, showed similar results. The results of western blot showed that the expression levels of claudin-1 and occludin in the infarcted hemisphere significantly decreased in the model group, compared with the ipsilateral hemispheres in the sham group $(P<0.01)$; however, the expression levels of claudin-1 and occludin were significantly increased in SLT administrated groups in 
comparison to the model group (all $\mathrm{P}<0.01$, Fig. $2 \mathrm{~b}$ ). Immunohistochemistry examination results showed that the expressions of claudin-1 and occludin in cerebral microvascular endothelial cells in the infarcted area were attenuated in the model group, in comparison to the same positions in the sham group; However, these down-regulations in claudin- 1 and occludin expressions were prevented by SLT treatments (Fig.2c).

Collectively, these results hint that the cerebral blood microvessels may be a key target of SLT, and the protection on them may constitute the foundation for SLT's therapeutic effects on brain ischemia.

\section{SLT activated the Nrf2/HO-1 pathway in the brain of MCAO rats, hinting that this pathway may participate in SLT's anti-brain ischemia effect}

The nuclear contents of Nrf2 in the infarcted hemispheres in the model group were significantly lower than that in the ipsilateral hemispheres in the sham group $(P<0.01)$, correspondingly Nrf2 contents in the cytoplasm in the model group was significantly higher $(P<0.01)$. This result indicates that after MCAO, Nrf2 pathway was drastically compromised in rat brains. However, SLT treatments rescued this fall down; in both SLT-L and SLT-H groups, Nrf2 levels in the nucleus were significantly increased $(P<0.05$ and $\mathrm{P}<0.01$, respectively, compared with the model group), and correspondingly the contents of Nrf2 in the cytoplasm of these two groups were significantly lower than that of the model group $(P<0.05$ and $P<0.01$, respectively, Fig.3a,b,d). Besides, the total protein levels of Nrf2 between all the groups showed no significant difference, suggesting the changes in Nrf2 pathway were mainly derived from posttranslational modifications, which is also the major regulation fashion of Nrf2 pathway (Fig.3c).

The downstream of Nrf2 exhibited similar changes. Treatments with SLT increased the protein expressions of HO-1 in the ischemia hemisphere, especially with SLT-H, which showed a significance in statistics (compared with the model group, $\mathrm{P}<0.01$, Fig. $3 \mathrm{e}$ ). Immunohistochemistry examination revealed that the upregulation of HO-1 by SLT treatments also happened in cerebral vascular endothelial cells (Fig. $3 \mathrm{~h}$ ). The activities of SOD and the contents of GSH in the infarcted hemispheres in the model group were also significantly decreased in comparison to the ipsilateral hemispheres in the sham group $(P<0.05$ in SOD activities, and P<0.05 in GSH contents); and these decreases were inhibited in SLT treatment groups as well, with better effects in SLT-H group, which has statistical significances in comparison to the model group ( $P<0.01$ in SOD activities, and $P<0.05$ in GSH contents, Fig. $4 f$ and $g$ ).

Taken together, these results showed SLT treatments activated Nrf2 pathway in the brain and imply that this pathway may play a key role in SLT's therapeutic effects on brain ischemia including the protection on cerebral microvascular endothelium.

\section{SLT protected hCMEC/D3 cells and activated Nrf2/HO-1 pathway in hCMEC/D3 cells in OGD/R injury}

The above results of in vivo experiments suggest that SLT may exert its anti-brain ischemia effect through cerebral microvascular protection, and the latter may be related to the activation of Nrf2 pathway. 
To confirm this assumption, we selected a human brain microvascular endothelial cell line, hCMEC/D3, and observed the direct effect of SLT on it.

Firstly, we observed the safety range of SLT. In the concentration range of $3.15-100 \mathrm{mg} / \mathrm{L}$, SLT did not significantly decrease the cell viabilities, on the contrary, at concentrations of 25,50 and $100 \mathrm{mg} / \mathrm{L}$, SLT significantly increased the cell viabilities ( $P<0.01$, compared with the normal group; Fig.4a). This result indicates that up to $100 \mathrm{mg} / \mathrm{L}$, SLT has no toxicities on hCMEC/D3 cells.

Then, we duplicated OGD/R model in hCMEC/D3 cells, which simulates ischemia injury in vivo, and treated the cells with SLT. The results showed that OGD/R injury significantly decreased the cell viabilities ( $P<0.01$, compared with the mock group); however, SLT treatments at concentrations of $3.125,6.25$ and $12.5 \mathrm{mg} / \mathrm{L}$ significantly increased the cell viabilities (all $\mathrm{P}<0.01$, compared with the model group, Fig.4b), confirming that SLT has a direct protective effect on microvascular endothelial cells against ischemia assault.

Thirdly, we investigated the activities of Nrf2 pathway in SLT's intervening. We selected the medium effective concentration, i.e. $6.25 \mathrm{mg} / \mathrm{L}$, of SLT to treat hCMEC/D3 cells. The results of immunocytofluorescence examinations showed that cells in the mock group exhibited weak expressions of Nrf2 and HO-1, in contrast, cells in the model group displayed slightly enhanced expressions; however, the expressions of these two proteins in cells of SLT treated groups were strongly enhanced in comparison to the model group, furthermore the enhancedly expressed Nrf2 was mainly nuclear distributed (Fig.4c,d). SOD activities and GSH levels in hCMEC/D3 cells showed an accordant situation. They were significantly decreased in OGD/R group ( $P<0.01$, compared with the mock group), however, compared with the model group, the SOD activities and GSH levels in SLT treated group were significantly increased $(P<0.01$, Fig.4e,f). This result directly testified that SLT treatment activates Nrf2 pathway in microvascular endothelial cells and further links Nrf2 pathway to SLT's vascular endothelium protection.

\section{Blocking of Nrf2 pathway by siRNA interference inhibited SLT's protective effect against OGD/R in hCMEC/D3 cells, finally confirming the obligatory role of Nrf2 pathway in microvascular endothelium protection and anti-brain ischemia effects of SLT}

To finally confirm the role of Nrf2 pathway in SLT's therapeutic effect, we performed a counter-evidence experiment.

Firstly, we constituted siRNAs to knock down Nrf2. The optimal transfection concentration that lead to minimum cytotoxicity and can efficiently downregulate target gene expressions was determined (Fig.5A). Then, three Nrf2 siRNA (siRNA-1, siRNA-2, siRNA-3) and negative control siRNA (NC siRNA) sequences were designed, synthesized and transfected to hCMEC/D3 cells. The results showed that Nrf2 siRNA-3 had the strongest ability to inhibit Nrf2 protein expression, and interestingly, this siRNA also significantly decreased the cell viability in OGD/R injury, which is agreed with the well recognized safeguard role of Nrf2 pathway in ischemia injury ( $P<0.01$, compared with NC siRNA+OGD/R group, Fig.5B,C,D). Therefore, 
Nrf2 siRNA-3 was selected to study the role of Nrf2 in SLT's therapeutic effect on OGD/R injury of hCMEC/D3 cells.

We next duplicated OGD/R injury in hCMEC/D3 cells, treated it with SLT, and observed the blocking effect of Nrf2 siRNA on SLT's therapy. As expected, the elevations of protein expressions of Nrf2 and HO-1 by SLT treatment $(\mathrm{P}<0.05$ or $0.01, \mathrm{NC}$ siRNA+OGD/R+SLT group vs. NC siRNA+OGD/R group) were significantly prevented by Nrf2 siRNA interference, indicating Nrf2 pathway was specifically blocked (all $\mathrm{P}<0.01,0 G D / \mathrm{R}+\mathrm{SLT}+\mathrm{Nrf2}$ siRNA group vs. NC siRNA+OGD/R+SLT group, Fig.6a). The alleviating effects of SLT on OGD/R injury in hCMEC/D3 cells include significantly increasing the cell viabilities, significantly reducing endothelium permeability, and significantly elevating expressions of claudin-1 and occludin $(\mathrm{P}<0.05$ or $0.01, \mathrm{NC}$ siRNA+OGD/R+SLT group vs. NC siRNA+OGD/R group, Fig.6b,c); however, these protective effects were all significantly inhibited by Nrf2 siRNA interference $(P<0.05$ or $P<0.01$, OGD/R+SLT+Nrf2 siRNA group vs. NC siRNA+OGD/R+SLT group; and P>0.05, OGD/R+Nrf2 siRNA group vs. OGD/R+ SLT+Nrf2 siRNA group; Fig.6b,c). This result undoubtedly indicates the protective effect of SLT on OGD/R injury of hCMEC/D3 cells is Nrf2 pathway dependent.

\section{Discussion}

In the present study, we showed that: (『) SLT alleviated cerebral ischemia and the subsequent injuries in neurological functions in rats; ( $\nabla)$ SLT attenuated ischemia induced brain edema and cerebral

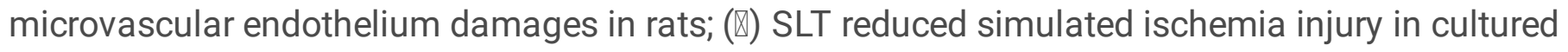
microvascular endothelial cells; ( $\mathbb{\nabla}$ ) the protective effect of SLT on microvascular endothelial cells was dependent on Nrf2 signaling pathway.

The mass and devastating neuron damages are the ultimate consequence of cerebral infarction, however, with the deepening of the research on mechanism of brain ischemia, factors out of the neural cells deserve more and more attentions [25]. Ischemia leads to a drastic drop in the supplies of oxygen and nutrients mainly including glucose, which injures neurons and also injures brain vascular endothelial cells at the same time [26]. The vascular endothelial cells exhibit severe cellular edema, i.e. hydropic degeneration, even death, which results in disruption in the endothelium TJs, and attenuates its barrier function, leading to serious exudations from the blood vessels, which finally cause grievous edema and inflammation in the brain tissue, generating additive injuries to the neurons [25]. These injures secondary to ischemia are far more detrimental, both more violent and more extensive, which could expand to semiischemia and even non-ischemia regions, thus significantly amplify the damages, even by fold [26]. Actually, most of the fatally devastating events elicited by cerebral infarction, such as cerebral edema, intracranial hypertension, cerebral hernia, coma, and even death are largely related to vascular damages [27]. Thus, the vascular factor is a pivotal role in the pathogenesis of cerebral ischemia, even more important than ischemia itself.

The formation of endothelial TJs is dependent on a sort of specific proteins, of which claudin and occludin are two major members. These two proteins have a similar action mode, that is the same kind 
molecules in membranes of the two neighboring cells combine each other and form homological complexes, thus seal off the intercellular gap, blocking the passing of blood contents [27]. Besides, these two proteins also have a protective effect on endothelial cells against hazardous stimuli. For example, knockdown of occludin potentiated cytokines secretion, inflammasome activation, and pyroptosis occurrence in TNF-a-treated bEnd.3 cells [28].

It should be noted that claudin has some subtypes, however, it is acknowledged that claudin-1 is the main type of that expressed in the brain blood vessels. Berndt et al. reported that claudin-1 was expressed in human brain capillaries as the strongest claudin, even significantly more than claudin-5 [29]. A recent study showed that claudin-1 replaced claudin- 5 at the TJs of brain capillary endothelial cells during the regeneration phase after stroke [30]. Furthermore, knockout of claudin-1 in null mice is lethal, suggesting its irreplaceable role for TJ preservation [31].

Cerebral ischemia induces a rapid degradation of claudin and occludin, which disrupts the TJ structure and renders the endothelial cells more vulnerable, accelerating their damages [32]. These finally result in the collapse of the blood vessel barrier and severe edema in brain [33]. In contrast, preventing the loss of claudin and occludin can alleviate brain edema and lesions [34, 35].

In the present study, the administrations of SLT significantly and dose-dependently reduced the infarct size, alleviated neural morphological injuries and improved neurological functions in rats $24 \mathrm{~h}$ after MCAO, indicating that SLT has an explicit protective effect against bran ischemia. Additionally, SLT treatments also significantly decreased the brain water content and elevated the expressions of endothelial TJ proteins including claudin-1 and occludin. This result is accordant with our previous reports, and further hints that SLT may exert its therapeutic effects on brain ischemia through protection on cerebral blood vessel endothelial cells [20-21].

Cerebral microvascular endothelium is highly vulnerable to oxidative stress. It has been suggested that the disruptions of blood vascular endothelial TJs and endothelial cells damages in brain infarction are all closely related to peroxidation [36,37].

Oxidative stress refers to a relative surplus of ROS, which is a sort of oxygen molecule derivatives having an extremely vivacious oxidative activity and mainly includes super oxygen anions, hydroxyl radicals, and hydrogen peroxides. The origin of ROS in endothelial cells includes the mitochondrial electronic transition chain, the cytoplasm hypoxanthine-xanthine oxidase system, and NAPDH oxidase system, etc [38]. Usually, super oxygen anions are firstly generated, and are then soon metabolized into hydroxyl radicals and hydrogen peroxides, which even have more strong oxidative abilities.

The targets of ROS are mainly biological macromolecules including DNA both in the nucleus and in the mitochondria, cytomembrane lipids and proteins. ROS breaks the unsaturated links in them, leading to severe abnormalities in their structures and functions, which further cause disorders in cellular signal transductions and organelle functions, even generate ceases in pivotal physiological procedures such as 
energy metabolism, cellular structure repairs, and eliminations of damaged organelles, etc., and finally result in cell degenerations even cell death [39].

Nrf2 pathway is the major anti-oxidation system in most cell types including the blood vascular endothelial cells. Nrf2 is a nuclear transcription factor, however in quiescent state, most of which are detained in the cytoplasm and face to ubiquitin mediated degradation [40]. Upon oxidative assaults, Nrf2 is released. The freed Nrf2 then translocates into the nucleus and combines with DNA, promoting the transcriptions of a series of anti-oxidation genes including HO-1, SOD, and GST-1, etc. [41,42]. HO-1 catalyzes the generation of carbon monoxide, which has a strong reduction ability; SOD degrades the super oxygen anions; and GST-1 promotes the generation of GSH, which is a powerful and broad spectrum ROS scavenger. Thus with these potent downstream effectors, Nrf2 pathway exerts an efficient elimination on free radicals, making a mighty counterattack to the oxidation assault.

However, interestingly, this passive defense system can play active roles in some conditions. In the ischemia preconditioning, small amount ROS generated from a transient ischemia-reperfusion can activate Nrf2 pathway, endowing the cells a strong antioxidation ability to confront the following severe ischemia challenge [10,12]. Another example is the preconditioning-like effects elicited by some chemicals, which have strong redox activities and can liberate Nrf2 in the similar way with ROS [43,44]. Many natural substances have this effect, such as some dietary phytochemicals including epicatechin, catechin, quercetin, and kaempferol, etc.; more importantly, numerous Chinese herbal ingredients are also included, among which the famous ones comprise salviolic acids, ligustrazine, and luteolin, etc. Thus the Nrf2 pathway forms a bridge for natural compounds to exert the physical modulatory effects $[13,45-48]$.

In consideration of the key role of peroxidation in vascular endothelium damages in ischemia and the key role of Nrf2 in antioxidation,

otent a effectors xygen anions GSH were all increased by SLT tigateed

we next investigated the role of Nrf2 pathway in SLT's endothelial protective effects. In the in vivo experiments, SLT treatments significantly elevated the function of Nrf2 in the brain in infarction; accordingly, the expression of HO-1, activity of SOD and content of GSH in the brain were all increased by SLT, indicating the downstreams of Nfr2 were activated. Notably, the immunochemistry examination positioned this Nrf2 pathway activation to cerebral vascular endothelial cells. These data preliminarily demonstrate the central role of Nrf2 pathway in SLT's vascular endothelium protection.

To obtain the direct evidence, we performed an in vitro experiment. We selected hCMEC/D3, a human brain microvascular endothelial cell line as the experimental material. Interestingly, this cell line was found to express claudin- 1 as one of the most dominant claudin subtypes, which is similar to the situation of in vivo brain vascular endothelial cells [24-26]. And we selected OGD/R model, in which the cells are incubated with glucose free medium in an anoxia circumstance to simulate ischemia in vivo, and are then returned to normal medium and circumstance to simulate reperfusion. 
At first, SLT significantly inhibited OGD/R induced drop in viabilities of hCMEC/D3 cells, confirming its direct protective effects on brain vascular endothelial cells against ischemia. Moreover, the Nrf2 pathway was also activated in hCMEC/D3 cells by SLT treatments, further revealing the relationship between Nrf2 pathway and SLT's therapeutic effects. Lastly, we used siRNA to block the function of Nrf2, and expectedly, the protective effects of SLT on hCMEC/D3 cells against OGD/R injury were all diminished, conclusively confirming the obligatory role of Nrf2 pathway in SLT's protection on brain vascular endothelium. Furthermore, combining the results of in vivo and in vitro experiments, it can be inferred that the Nrf2 pathway dependent protection on cerebral microvascular endothelial cells underlies the therapeutic effect of SLT on brain ischemia (the putative mechanism of SLT's anti-brain ischemia effect is shown in figure 7).

However, as a redox active drug, SLT also has the ability to directly scavenge ROS, then does this activity play a role in SLT's effects? As shown in our experiment results, the knockdown of Nrf2 expression resulted in a nearly total ablation in SLT's protective effects on hCMEC/D3 cells, thus it indicates that the direct antioxidation effect of SLT does not make a significant contribution to its blood vascular endothelium protection in brain ischemia.

At last, an interesting issue should be discussed. SLT has multiple ingredients, then which exert this Nrf2 dependent therapeutic effect? The main ingredients of SLT include ginsenosides, flavones, ginkgolides and crocin (see table1). Although according to the published studies, all of them have active redox characteristics and the potential to activate Nrf2, there exit differences in their activities. Flavones and crocin possessed the strongest redox activities, which are far more than that of the others $[49,50]$. Additionally, their amounts occupy nearly a half of that of SLT's main active ingredients. Thus it seems that flavones and crocin in SLT may be the main active ingredients for the Nrf2 dependent endothelium protection in brain ischemia. However, this is only a conjecture, what are the exact roles of SLT's ingredients in its therapy, and what are the relationships between them, simple additive relation, or synergic relation, or even antagonistic relation, need to be further studied in the future.

\section{Conclusions}

Data of this study suggest that SLT's therapeutic effect against brain ischemia is related to its protection on the cerebral vascular endothelial cells, which is dependent on the activation of Nrf2 antioxidation pathway. This study may have significant meanings for elucidating the therapeutic mechanism of SLT on stroke, and may be also meaningful for searching effective remedies for stroke.

\section{Abbreviations}

SaiLuoTong capsule: SLT; cerebral blood vascular endothelial cells: CBVEC; brain blood barrier: BBB; tight junctions: TJs; reactive oxygen species: ROS; nuclear factor erythroid 2-related factor 2: Nrf2; acetylcholine: ach; permanent middle cerebral artery occlusion: pMCAO; 2,3,5-Triphenyltetrazolium chloride: TTC; heme oxygenase-1: HO-1; horseradish peroxidase: HRP; superoxide dismutase: SOD; 
glutathione: GSH; human brain microvascular endothelial cell line: hCMEC/D3; Oxygen-glucose deprivation and reoxygenation (OGD/R); fluorescein isothiocyanate: FITC.

\section{Declarations}

\section{Acknowledgements}

The authors acknowledge the Department of Pathology of Xiyuan Hospital, China Academy of Chinese Medical Science for providing helpful advice. Research was supported by grants from Beijing Natural Science Foundation (7194314) and grants from China Natural Science Foundation (81973594).

\section{Authors' contributions}

FXD and XL conceived and coordinated the study. FXD and LP designed the experiments and wrote the paper. All authors performed the experiments in vitro and vivo. FXD, YB and WGR analyzed the data. FXD and LP reviewed and edited the manuscript. All authors read and approved the final manuscript.

\section{Funding}

This work was supported by Beijing Natural Science Foundation (7194314), National Science and Technology Major Projects (2018ZX09737-009) and National Natural Science Foundation of China (81973594), MiaoPu Funding of Xiyuan Hospital (2019XYMP-11).

\section{Availability of data and materials}

All data generated or analyzed during this study are included in this published article [and its supplementary information files].

\section{Ethics approval and consent to participate}

All animal experiments followed the Institutional Animal Care and all procedures were performed under the specifications set by the Ethics Committee of Xi Yuan Hospital of China Academy of Chinese Medical Sciences (Protocol No. 2019XLC015-2).

\section{Consent for publication}

All the authors have approved the manuscript.

\section{Competing interests}

The authors declare that they have no competing interests.

\section{Author details}


${ }^{1}$ Institute of Basic Medical Sciences, Xiyuan Hospital of China Academy of Chinese Medical Sciences, No.1 Xiyuan Caochang, Haidian District, Beijing, 100091, China

${ }^{2}$ Key Laboratory of Pharmacology of Chinese Materia Medica, No.1 Xiyuan Caochang, Haidian District, Beijing, 100091, China

${ }^{3}$ Xiyuan Hospital of China Academy of Chinese Medical Sciences, the Department of Pathology, No.1 Xiyuan Caochang, Haidian District, Beijing, 100091, China

\section{References}

1. Donnan GA, Fisher M, Macleod M, Davis SM. Stroke Lancet. 2008;371:1612-3.

2. Bazzoni G, Dejana E. Endothelial cell-to-cell junctions: molecular organization and role in vascular homeostasis. Physiol Rev. 2004;84(3):869-901.

3. Abbott NJ, Rönnbäck L, Hansson E. Astrocyte-endothelial interactions at the blood-brain barrier. Nat Rev Neurosci. 2006; 7(1):41-53.

4. Obermeier B, Daneman R, Ransohoff RM. Development, maintenance and disruption of the bloodbrain barrier. Nat Med. 2013;19(12):1584-96.

5. Jiao H, Wang Z, Liu Y, Wang P, Xue Y. Specific role of tight junction proteins claudin-5, occludin, and ZO-1 of the blood-brain barrier in a focal cerebral ischemic insult. J Mol Neurosci. 2011; 44(2):130-9.

6. Sladojevic N, Stamatovic SM, Johnson AM, Choi J, Hu A, Dithmer S, et al. Claudin-1-Dependent Destabilization of the Blood-Brain Barrier in Chronic Stroke. J Neurosci. 2019;39(4):743-57.

7. Chen $\mathrm{H}, \mathrm{He} Y$, Chen S, Qi S, Shen J. Therapeutic targets of oxidative/nitrosative stress and neuroinflammation in ischemic stroke: Applications for natural product efficacy with omics and systemic biology. Pharmacol Res. 2020;158:104877.

8. Kuźma E, Lourida I, Moore SF, Levine DA, Ukoumunne OC, Llewellyn DJ. Stroke and dementia risk: A systematic review and meta-analysis. Alzheimers Dement. 2018;14(11):1416-26.

9. Loboda A, Damulewicz M, Pyza E, Jozkowicz A, Dulak J. Role of Nrf2/HO-1 system in development, oxidative stress response and diseases: an evolutionarily conserved mechanism. Cell Mol Life Sci. 2016;73(17):3221-47.

10. Nguyen T, Nioi P, Pickett CB. The Nrf2-antioxidant response element signaling pathway and its activation by oxidative stress. J Biol Chem. 2009; 284(20):13291-5.

11. Zhao J, Moore AN, Redell JB, Dash PK. Enhancing expression of Nrf2-driven genes protects the blood brain barrier after brain injury. J Neurosci. 2007;27(38):10240-8.

12. Zhang R, Xu M, Wang Y, Xie F, Zhang G, Qin X. Nrf2-a promising therapeutic target for defensing against oxidative stress in stroke. Mol. Neurobiol.2017;54:6006-17.

13. Hu S, Wu Y, Zhao B, Hu H, Zhu B, Sun Z, et al. Panax notoginseng Saponins Protect Cerebral Microvascular Endothelial Cells against Oxygen-Glucose Deprivation/Reperfusion-Induced Barrier 
Dysfunction via Activation of PI3K/Akt/Nrf2 Antioxidant Signaling Pathway. Molecules. 2018;23(11):2781.

14. Li C, Wang R, Hu C, Wang H, Ma Q, Chen S, et al. Pyridoxine exerts antioxidant effects in cell model of Alzheimer's disease via the Nrf-2/HO-1 pathway. Cell Mol Biol. 2018;64:119-124.

15. Wang W, Wang X, Zhang XS, Liang CZ. Cryptotanshinone attenuates oxidative stress and inflammation through the regulation of Nrf-2 and NF-KB in mice with unilateral ureteral obstruction. Basic Clin Pharmacol Toxicol. 2018;123:714-20.

16. Liu J, Jiang Y, Zhang G, Lin Z, Du S. Protective effect of edaravone on blood-brain barrier by affecting NRF-2/HO-1 signaling pathway. Exp Ther Med. 2019;18(4):2437-42.

17. Li T, Wang H, Ding Y, Zhou M, Zhou X, Zhang X, et al. Genetic elimination of Nrf2 aggravates secondary complications except for vasospasm after experimental subarachnoid hemorrhage in mice. Brain Res. 2014;1558: 90-9.

18. Jia J, Wei C, Chen S, Li F, Tang Y, Qin W, et al. Efficacy and safety of the compound Chinese medicine SaiLuoTong in vascular dementia: A randomized clinical trial. Alzheimers Dement (NY). 2018;4:10817.

19. Steiner GZ, Bensoussan A, Liu J, Hohenberg MI, Chang DH. Study protocol for a randomised, doubleblind, placebo-controlled 12-week pilot phase II trial of Sailuotong (SLT) for cognitive function in older adults with mild cognitive impairment. Trials. 2018;19(1):522.

20. Seto SW, Chang D, Ko WM, Zhou X, Kiat H, Bensoussan A, et al. Sailuotong Prevents Hydrogen Peroxide (H囚OZ)-Induced Injury in EA.hy926 Cells. Int J Mol Sci. 2017;18(1):95.

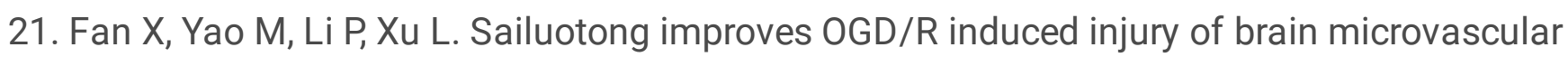
endothelial cells by up-regulating Nrf2/HO-1 signaling pathway. Chinese Pharmacological Bulletin. 2020;36(07):1018-23.

22. Zhang Y, Liu J, Yao M, Song W, Zheng Y, Xu L, et al. Sailuotong Capsule Prevents the Cerebral Ischaemia-Induced Neuroinflammation and Impairment of Recognition Memory through Inhibition of LCN2 Expression. Oxid Med Cell Longev. 2019;2019:8416105.

23. Xu L, Liu JX, Cong WH, Wei CE. Effects of Weinaokang capsule on intracephalic cholinergic system and capability of scavenging free radicas in chronic cerebral hypoperfusion rats. Zhongguo Zhong Yao Za Zhi. ; 33(5):531-4.

24. Bederson JB, Pitts LH, Tsuji M, Nishimura MC, Davis RL, Bartkowski H. Rat middle cerebral artery occlusion: evaluation of the model and development of a neurologic examination. Stroke. 1986; 17(3):472-6.

25. Andjelkovic AV, Xiang J, Stamatovic SM, Hua Y, Xi G, Wang MM, et al. Endothelial Targets in Stroke: Translating Animal Models to Human. Arterioscler Thromb Vasc Biol. 2019;39(11):2240-7.

26. Brouns R, De Deyn PP. The complexity of neurobiological processes in acute ischemic stroke. Clin Neurol Neurosurg. 2009;111(6):483-95.

27. Rahimi N. Defenders and Challengers of Endothelial Barrier Function. Front Immunol. 2017;8:1847. 
28. Zhang Y, Li X, Qiao S, Yang D, Li Z, Xu J, et al. Occludin degradation makes brain microvascular endothelial cells more vulnerable to reperfusion injury in vitro. J Neurochem. 2020; doi: 10.1111/jnc. 15102 .

29. Berndt P, Winkler L, Cording J, Breitkreuz-Korff O, Rex A, Dithmer S, et al. Tight junction proteins at the blood-brain barrier: far more than claudin-5. Cell Mol Life Sci. 2019;76(10):1987-2002.

30. Sladojevic N, Stamatovic SM, Johnson AM, Choi J, Hu A, Dithmer S, et al. Claudin-1-dependent destabilization of the blood-brain barrier in chronic stroke. J Neurosci. 2019;39(4):743-57.

31. Furuse M, Hata M, Furuse K, Yoshida Y, Haratake A, Sugitani Y, et al. Claudin-based tight junctions are crucial for the mammalian epidermal barrier: a lesson from claudin-1-deficient mice. J Cell Biol. 2002;156(6):1099-111.

32. Zhang X, Fan Z, Jin T. Crocin protects against cerebral- ischemia-induced damage in aged rats through maintaining the integrity of blood-brain barrier. Restor Neurol Neurosci. 2017;35(1):65-75.

33. Sandoval KE, Witt KA. Blood-brain barrier tight junction permeability and ischemic stroke. Neurobiol Dis. 2008;32(2):200-19.

34. Takenaga Y, Takagi N, Murotomi K, Tanonaka K, Takeo S. Inhibition of Src activity decreases tyrosine phosphorylation of occludin in brain capillaries and attenuates increase in permeability of the bloodbrain barrier after transient focal cerebral ischemia. J Cerebral Blood Flow Metab.2009; 29:1099-108.

35. Stamatovic SM, Johnson AM, Keep RF, Andjelkovic AV. Junctional proteins of the blood-brain barrier: New insights into function and dysfunction. Tissue Barriers. 2016;4(1):e1154641.

36. Freeman LR, Keller JN. Oxidative stress and cerebral endothelial cells: regulation of the blood-brainbarrier and antioxidant based interventions. Biochim Biophys Acta. 2012;1822:822-9.

37. Musch MW, Walsh-Reitz MM, Chang EB. Roles of ZO-1, occludin, and actin in oxidant-induced barrier disruption. Am J Physiol Gastrointest Liver Physiol.2006;290(2):G222-31.

38. Fraser PA. The role of free radical generation in increasing cerebrovascular Free Radic Biol Med. 2011;51(5):967-77.

39. Olmez I, Ozyurt H. Reactive oxygen species and ischemic cerebrovascular disease. Neurochem Int. 2012;60(2):208-12.

40. Itoh K, Wakabayashi N, Katoh Y, Ishii T, Igarashi K, Engel JD, et al. Keap1 represses nuclear activation of antioxidant responsive elements by Nrf2 through binding to the amino-terminal Neh2 domain. Genes Dev. 1999;13(1):76-86.

41. Harvey CJ, Thimmulappa RK, Singh A, Blake DJ, Ling G, Wakabayashi N, et al. Nrf2-regulated glutathione recycling independent of biosynthesis is critical for cell survival during oxidative stress. Free Radic Biol Med. 2009;46(4):443-53.

42. Otterbein LE, Choi AM. Heme oxygenase: colors of defense against cellular stress. Am J Physiol Lung Cell Mol Physiol. 2000;279(6):L1029-37.

43. Ashabi G, Khalaj L, Khodagholi F, Goudarzvand M, Sarkaki A. Pre-treatment with metformin activates Nrf2 antioxidant pathways and inhibits inflammatory responses through induction of AMPK after 
transient global cerebral ischemia. Metab Brain Dis. 2015;30(3):747-54.

44. Ding Y, Chen M, Wang M, Li Y, Wen A. Posttreatment with 11-Keto- $\beta$-Boswellic Acid Ameliorates Cerebral Ischemia-Reperfusion Injury: Nrf2/HO-1 Pathway as a Potential Mechanism. Mol Neurobiol. 2015;52(3):1430-9.

45. Yamagata K. Protective Effect of Epigallocatechin Gallate on Endothelial Disorders in Atherosclerosis. J Cardiovasc Pharmacol. 2020;75(4):292-8.

46. Farkhondeh T, Yazdi HS, Samarghandian S. The Protective Effects of Green Tea Catechins in the Management of Neurodegenerative Diseases: A Review. Curr Drug Discov Technol. 2019;16(1):57-65.

47. Li H, Wang P, Huang F, Jin J, Wu H, Zhang B, et al. Astragaloside IV protects blood-brain barrier integrity from LPS-induced disruption via activating Nrf2 antioxidant signaling pathway in mice. Toxicol Appl Pharmacol. 2018;340:58-66.

48. Alfieri A, Srivastava S, Siow RCM, Cash D, Modo M, Duchen MR, et al. Sulforaphane preconditioning of the Nrf2/HO-1 defense pathway protects the cerebral vasculature against blood-brain barrier disruption and neurological deficits in stroke.

49. Barbagallo I, Galvano F, Frigiola A, Cappello F, Riccioni G, Murabito P, et al. Potential therapeutic effects of natural heme oxygenase-1 inducers in cardiovascular diseases. Free Radic Biol Med. 2013;65:1012-22.

50. Ma Q, He X. Molecular basis of electrophilic and oxidative defense: promises and perils of Nrf2. Pharmacol Rev. 2012;64(4):1055-1081.

\section{Tables}

Table 1 The main active ingredients of SLT 


\begin{tabular}{|c|c|c|c|}
\hline $\begin{array}{c}\text { Active } \\
\text { ingredients }\end{array}$ & $\begin{array}{c}\text { Contents } \\
\text { (mg/capsule) }\end{array}$ & Chemical structures & Origins \\
\hline $\begin{array}{l}\text { Ginsenoside } \\
\text { Rg1 }\end{array}$ & 2.4 & & \\
\hline $\begin{array}{c}\text { Ginsenoside } \\
\operatorname{Re}\end{array}$ & 1.6 & & $\begin{array}{l}\text { the roots of Panax } \\
\text { ginseng (ginseng) }\end{array}$ \\
\hline $\begin{array}{l}\text { Ginsenoside } \\
\text { Rb1 }\end{array}$ & 4.5 & & \\
\hline quercetin & 2.7 & & \\
\hline kaempferide & 2.3 & & \\
\hline isorhamnetin & 1.6 & & the leaves of Ginkgo \\
\hline $\begin{array}{c}\text { Ginkgolide } \\
\mathrm{A}\end{array}$ & 0.8 & & \\
\hline Ginkgolide B & 0.6 & & \\
\hline
\end{tabular}

The main active ingredients of SLT are shown in the table, data are obtained from reference[15].

Figures 
a

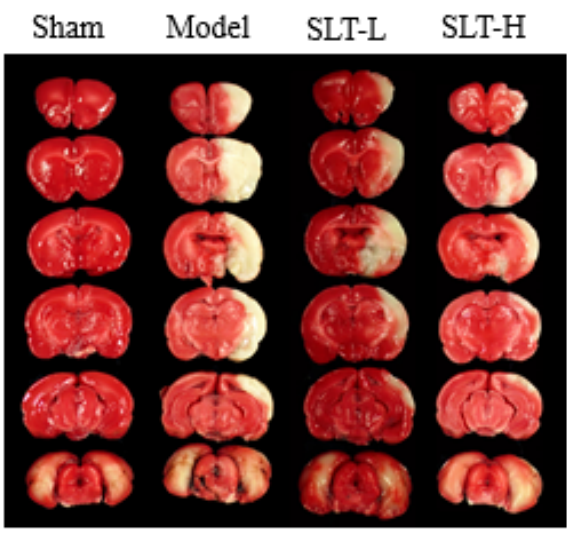

c

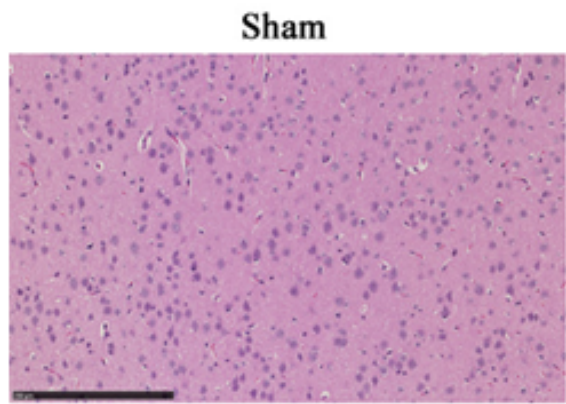

SLT-L

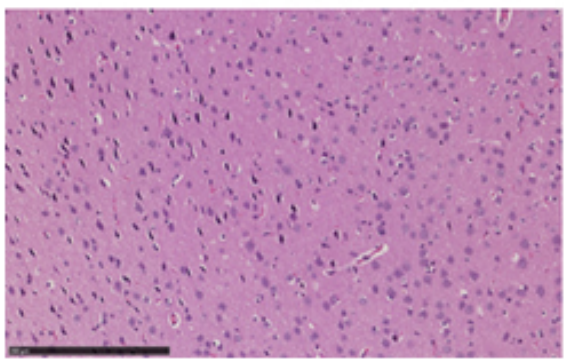

b

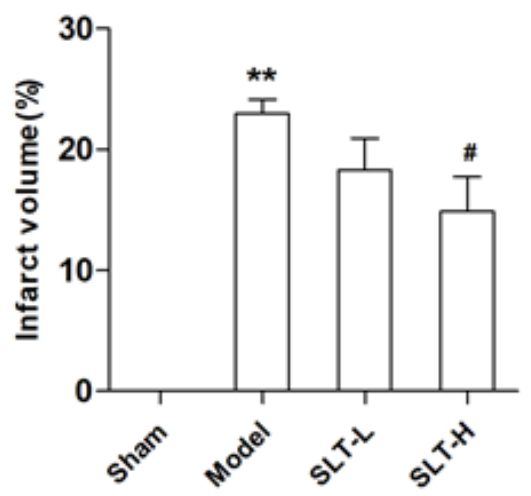

Model

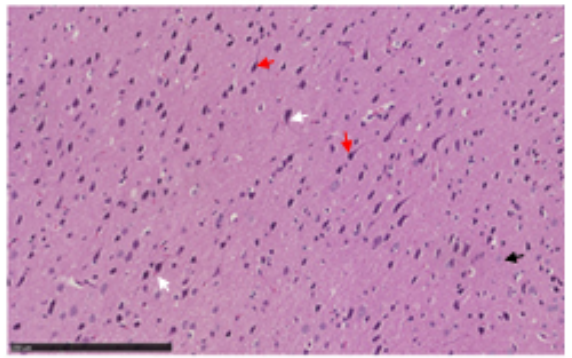

SLT-H

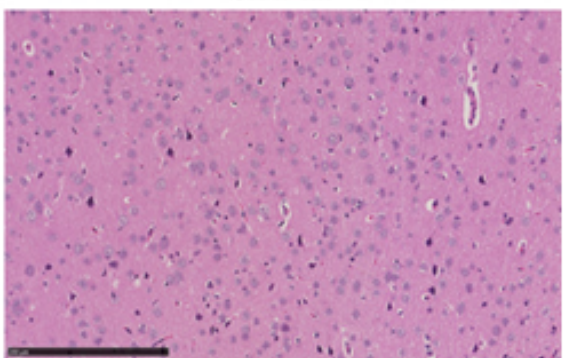

d

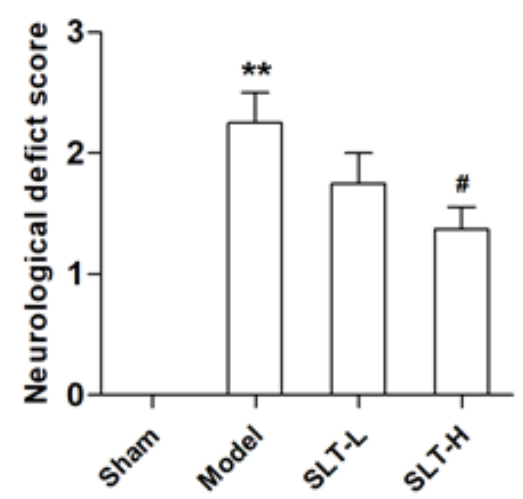

\section{Figure 1}

SLT attenuated cerebral infarction and neurological deficit in rats undergone MCAO. 24h after MCAO, the rats were neurologically tested, then were killed, and their brains were harvested for TTC and HE stainings. (a) Representative images of TTC staining. The white regions are the infarct tissues, while the red regions are the viable tissues. (b) Infarct volumes of the brains $(n=8)$. (c) Representative images of HE staining of the brain cortex in all the groups. The ischemic brain region in the model group presented 
severe neuronal loss and neuron degenerations, including cytoplasm acidophilic degeneration (indicated with white arrows), nuclear pyknosis (indicated with red arrows) and hydropic degeneration (indicated with black arrow). The neuronal damages were alleviated in SLT-L and SLT-H groups. Scale bar in all panels $=250 \mu \mathrm{m}$. (d) Neurological deficit scores of all the groups $(n=8)$. Data are expressed as mean $\pm S E M .{ }^{*} p<0.05, * \star p<0.01$, in comparison to the sham group; $\# p<0.05$, in comparison to the model group.

a

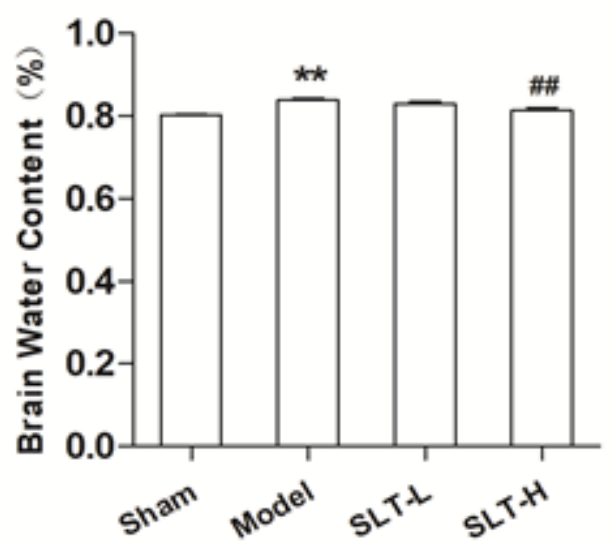

$\mathrm{b}$
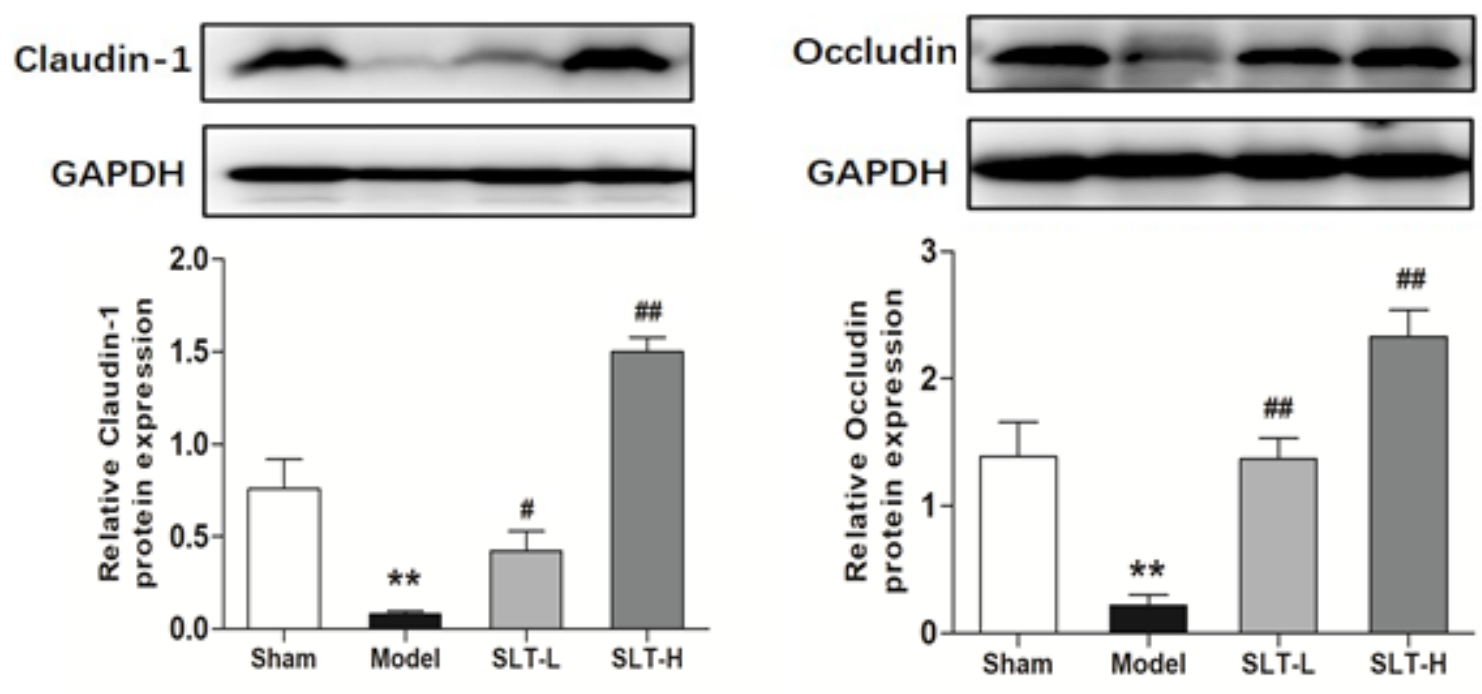

c
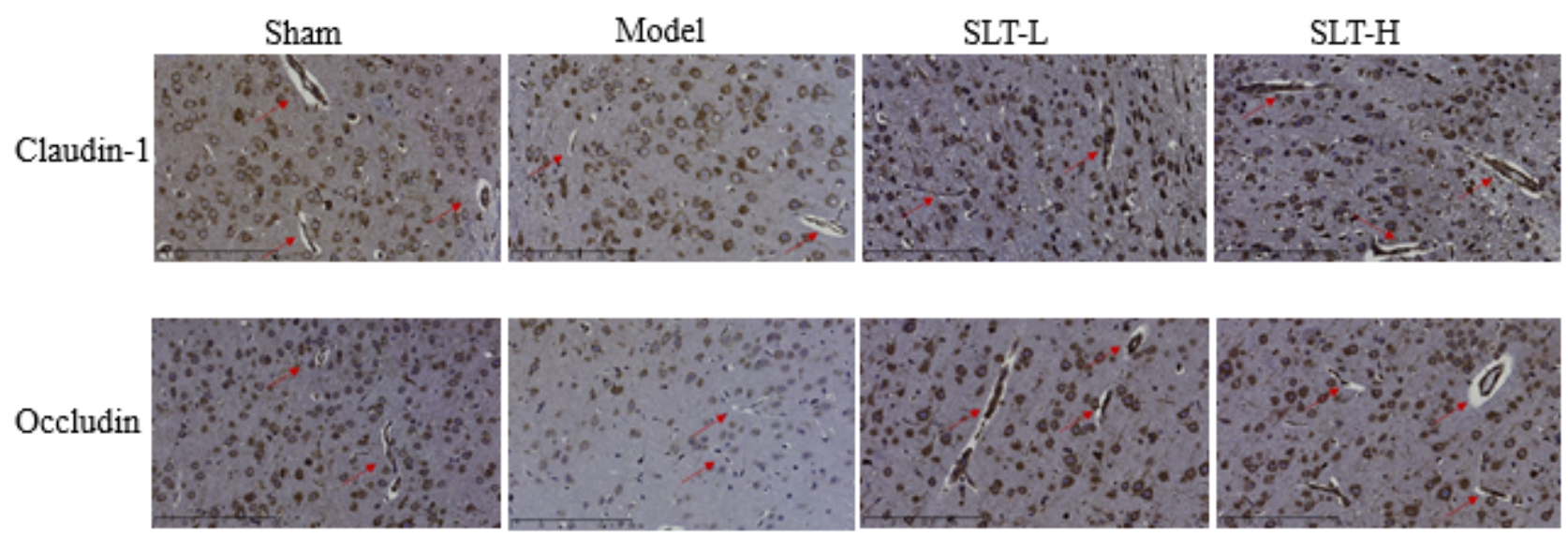

Figure 2 
SLT decreased brain edema and prevented the loss of tight junction proteins in cerebral blood vascular endothelium in MCAO rats. (a) Brain water contents of the ischemic hemispheres measured by the wet and dry weight method $(n=7)$. (b) Western blot assays for claudin- 1 and occludin expressions in the ischemic hemispheres $(n=3)$. (c) Representative images of immunohistochemistry examinations for claudin-1 and occludin in the ischemia areas. The arrows point to the blood vessels. Scale bar $=200 \mu \mathrm{m}$. Data are represented as mean $\pm S E M$. ${ }^{*} p<0.05,{ }^{*} p<0.01$, compared with the sham group; $\# p<0.05$, $\#$ \# $<0.01$, compared with the model group.

a
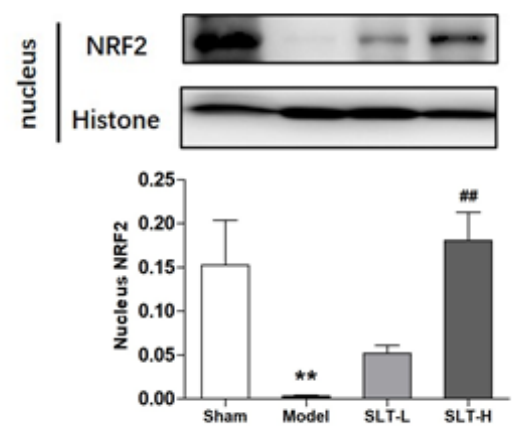

c

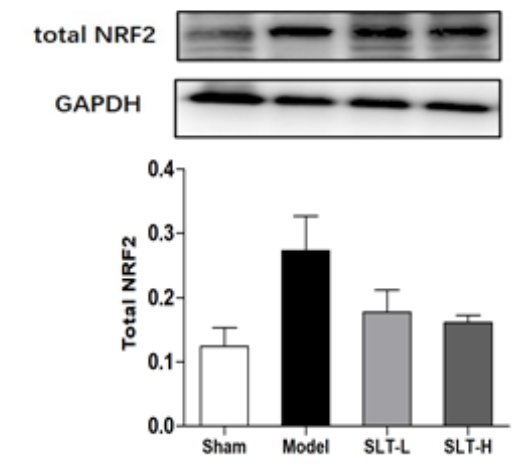

$\mathrm{e}$

HO-1

GAPDH

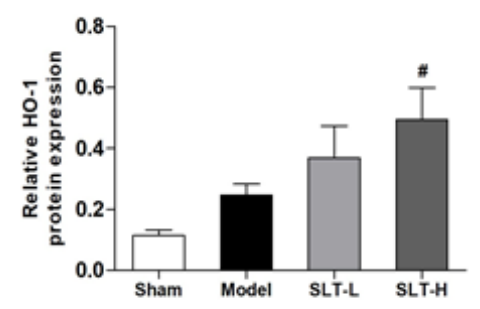

b
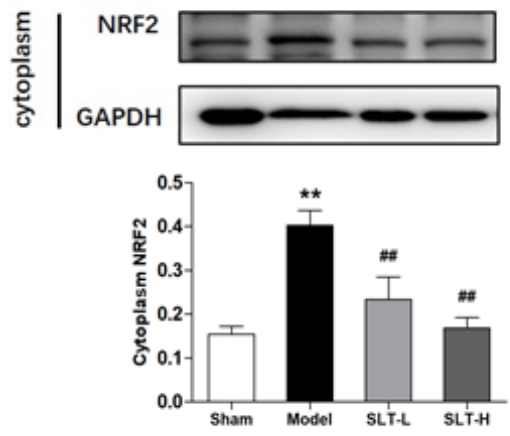

d

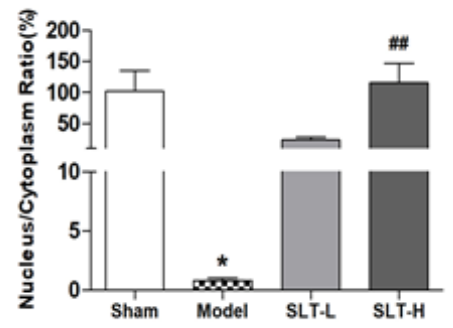

f

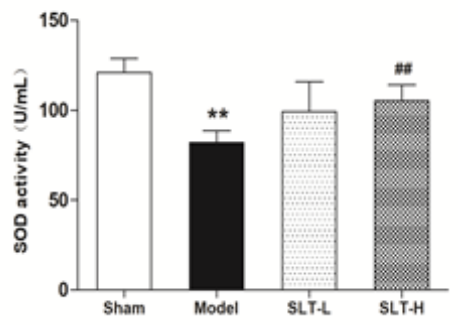

g

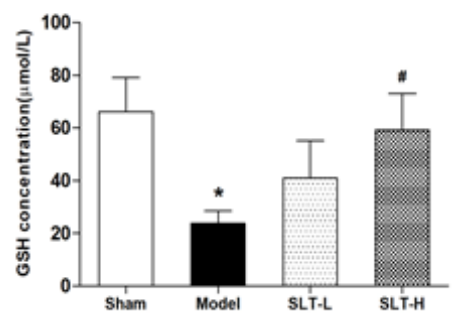

$\mathrm{h}$

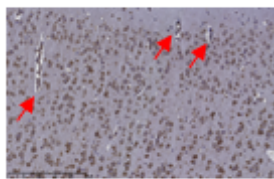

Sham

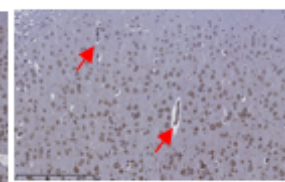

Model

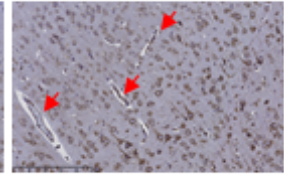

SLT-L

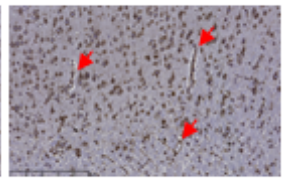

SLT-H 


\section{Figure 3}

SLT activated the Nrf2/HO-1 pathway in the brain after ischemia. (a), (b) and (c) Western blot assays for the protein levels in the ischemic hemispheres of nucleus Nrf2, cytoplasm Nrf2, and the total Nrf2, respectively $(n=3)$. (d) The ratio of Nrf2 in the nucleus to that in the cytoplasm, the results were calculated from data in (a) and (b). (e) HO-1 expressions in the ischemic hemispheres. (f) and (g) SOD activities and GSH contents in the ischemic hemispheres, respectively $(n=8)$. (h) Immunochemistry examination for HO1 expressions in the ischemic regions. Red arrows indicate the blood vessels. Scale bar in all panels $=250$ $\mu \mathrm{m}$. Data are expressed as mean $\pm S E M$. ${ }^{*} p<0.05,{ }^{*} p<0.01$ vs. the Sham group; $\# p<0.05, \# \# p<0.01$ vs. the Model group. 

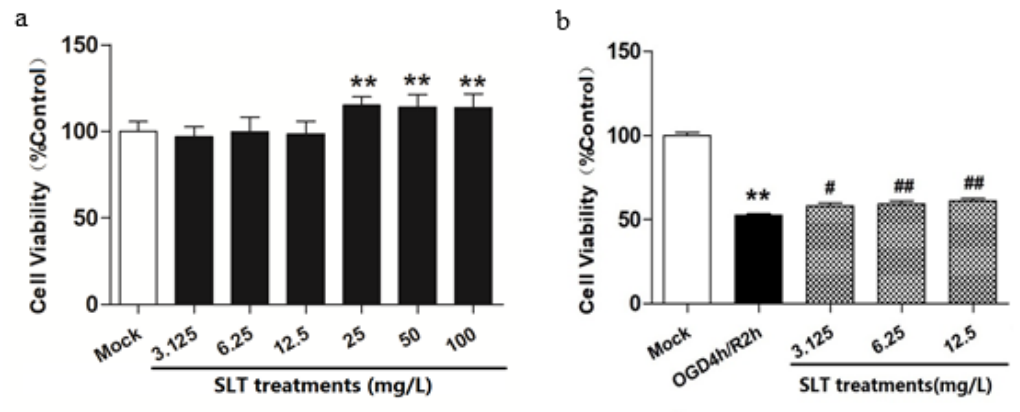

c
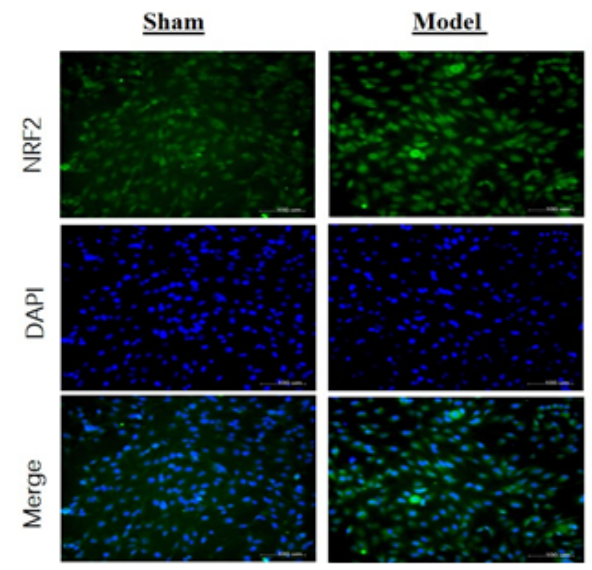

$\underline{\text { SLT }}$
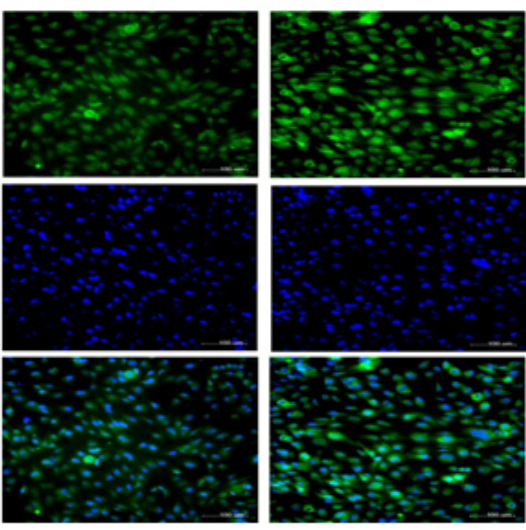

$\mathrm{d}$
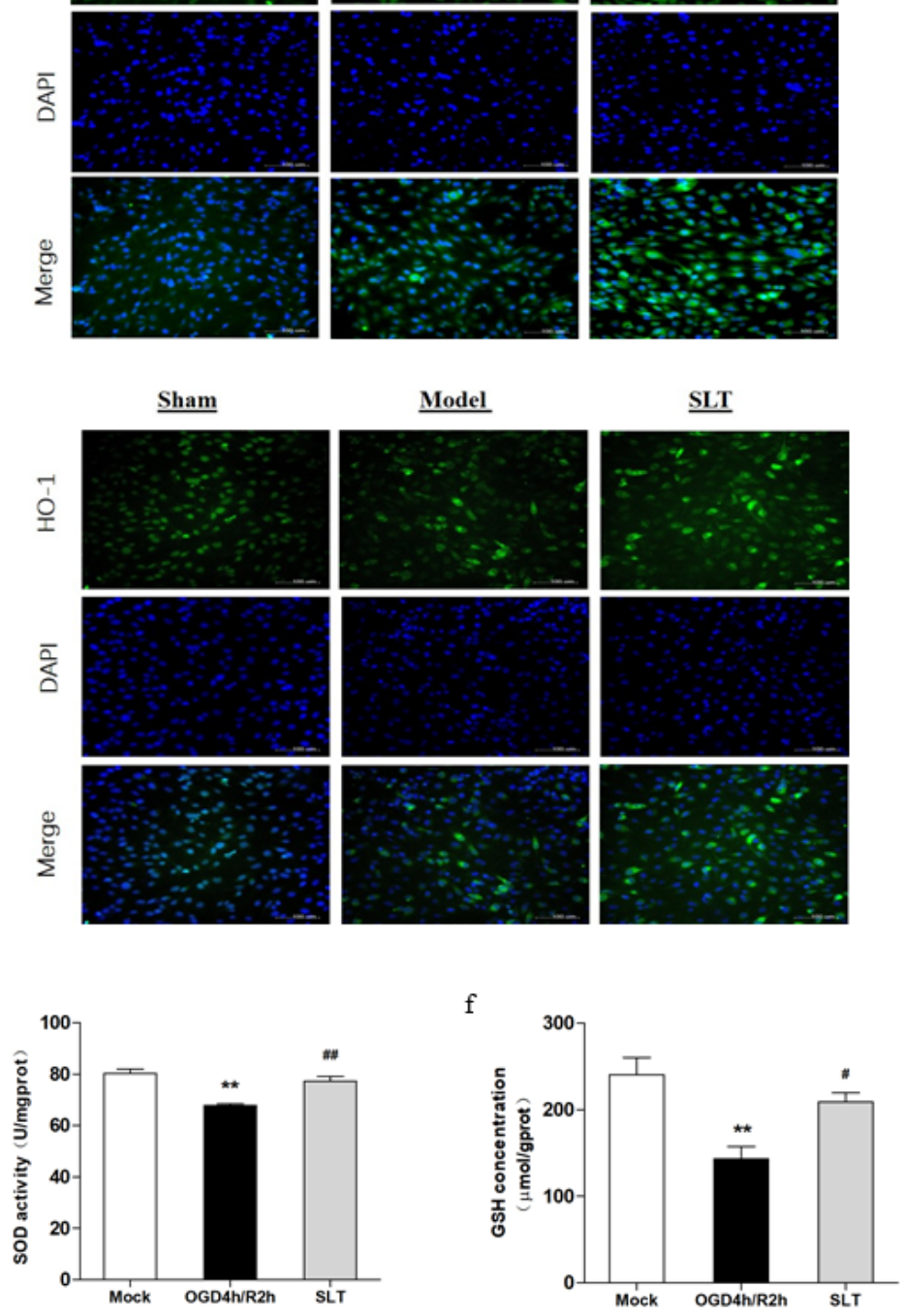

\section{Figure 4}

SLT alleviated OGD/R injury and activated Nrf2/HO-1 pathway in hCMEC/D3 cells. (a) Toxicity test of SLT on hCMEC/D3 cells $(n=6)$. The cells were cultured with different concentrations of SLT $(3.125,6.25,12.5$, 25,50 , and $100 \mathrm{mg} / \mathrm{L}$ ) for $24 \mathrm{~h}$ and the cell viabilities were examined by CCK-8 method. (b) The therapeutic effects of SLT on OGD/R injury in hCMEC/D3 cells $(n=6)$. (c) and (d), images of cytoimmunofluorescence examinations for Nrf2 and HO-1 expressions in cells undergone OGD/R injury, 
respectively. Scale bar $=100 \mu \mathrm{m}$. (e) and (f) SOD activities and GSH contents in hCMEC/D3 cells undergone OGD/R injury, respectively $\left(n=5\right.$ each). Data are expressed as mean $\pm S E M .{ }^{*} p<0.05,{ }^{* *} p<0.01$ vs. the Mock group; $\# p<0.05, \# \# p<0.01$ vs. the OGD/R group.

A
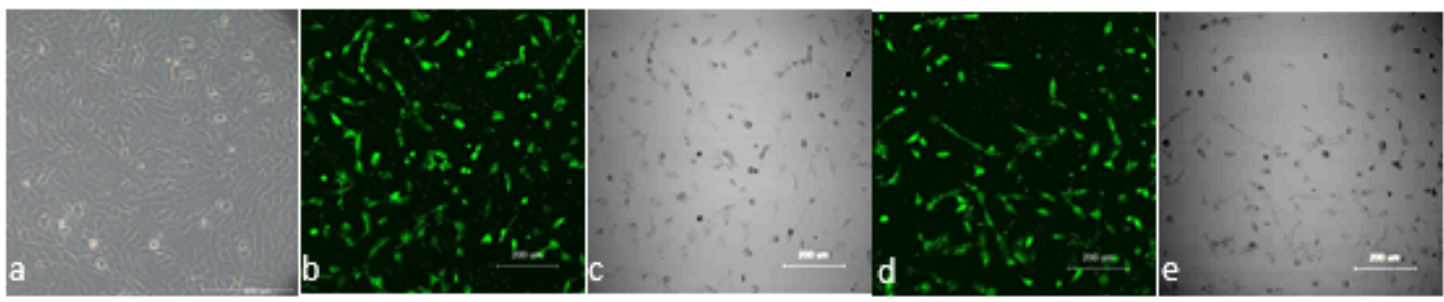

B

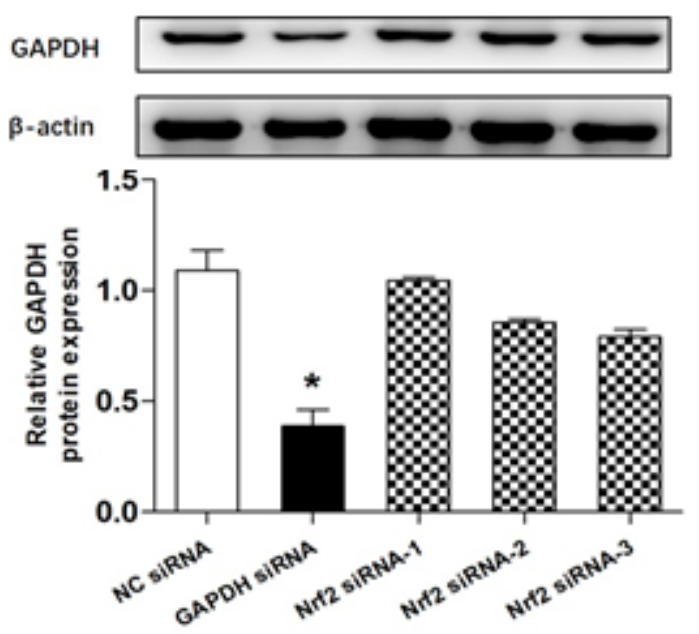

$\mathrm{C}$

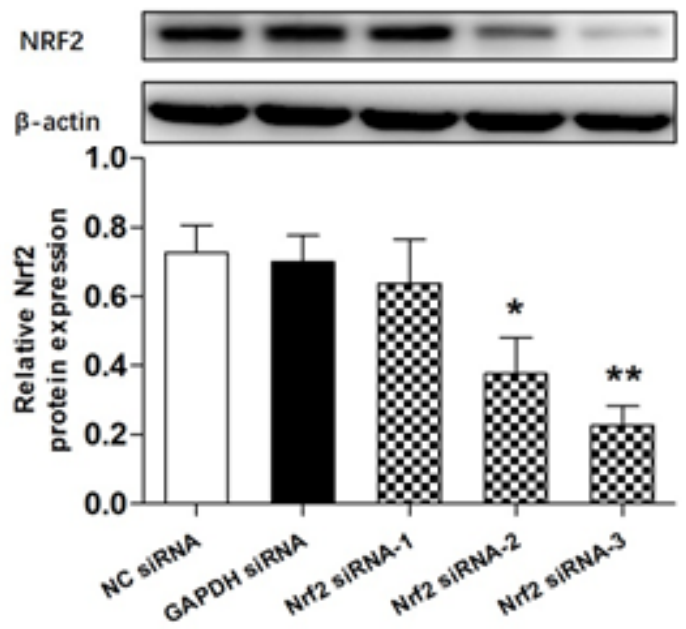

$\mathrm{D}$

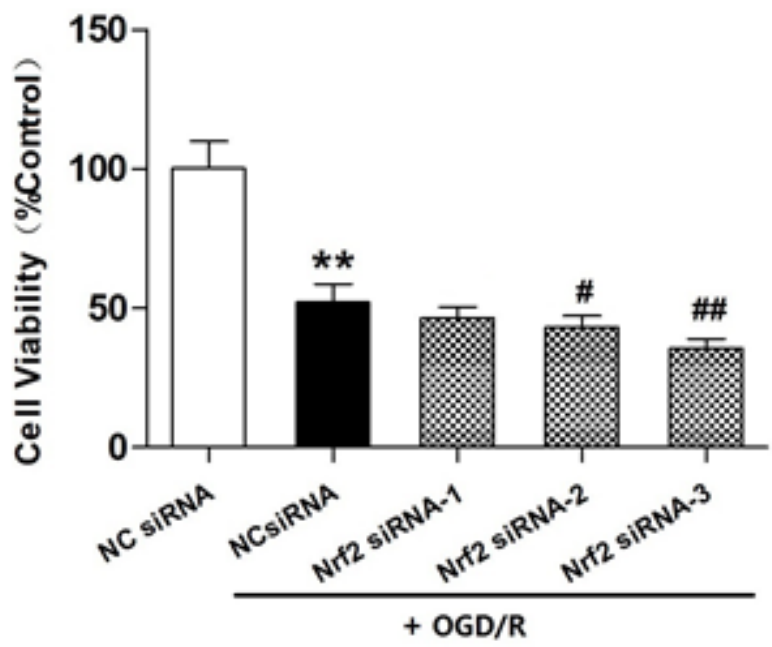

Figure 5

The effects of different Nrf2 siRNA interferences on Nrf2 expressions and hCMEC/D3 cell viabilities in OGD/R. (A) (a) hCMEC/D3 cells in normal culture condition. (b,c,d,e) Different concentrations of the negative control siRNA labeled with green fluorescence were treated to hCMEC/D3 cells to help optimize 
the transfection conditions. (b) and (c), fluorescence and bright field images of hCMEC/D3 cells treated with $50 \mathrm{nmol} / \mathrm{L}$ plasmid, respectively. (d) and (e), fluorescence and bright field images of of hCMEC/D3 cells treated with $100 \mathrm{nmol} / \mathrm{L}$ plasmid, respectively. Plasmids of the two concentrations showed a similar transfection efficiency, while the lower one showed less cell loss, thus $50 \mathrm{nmol} / \mathrm{L}$ was selected as the optimal concentration for transfection and was used in the subsequent experiments. (B) and (C) Western blot assays for expressions of GAPDH and Nrf2 in hCMEC/D3 cells, respectively. The cells were transfected with GAPDH siRNA plasmid or Nrf2 siRNA plasmids (Nrf2 siRNA-1, Nrf2 siRNA-2 and Nrf2 siRNA-3); the results showed the expressions of target genes were specifically knockdown by aiming siRNAs, testifying the specificities and efficiencies of the siRNAs. Besides, Nrf2 siRNA-3 showed the strongest ability to interfere with the expression of Nrf2. (D) The effects of different Nrf2 siRNAs on $\mathrm{OGD} / \mathrm{R}$ injury of hCMEC/D3 cells. Data are expressed as mean \pm SEM $(n=3) .{ }^{*} p<0.05,{ }^{\star \star} p<0.01$ vs. negative control siRNA (NC siRNA); \#p<0.05, \#\#p<0.01 vs. NC siRNA+OGD/R.
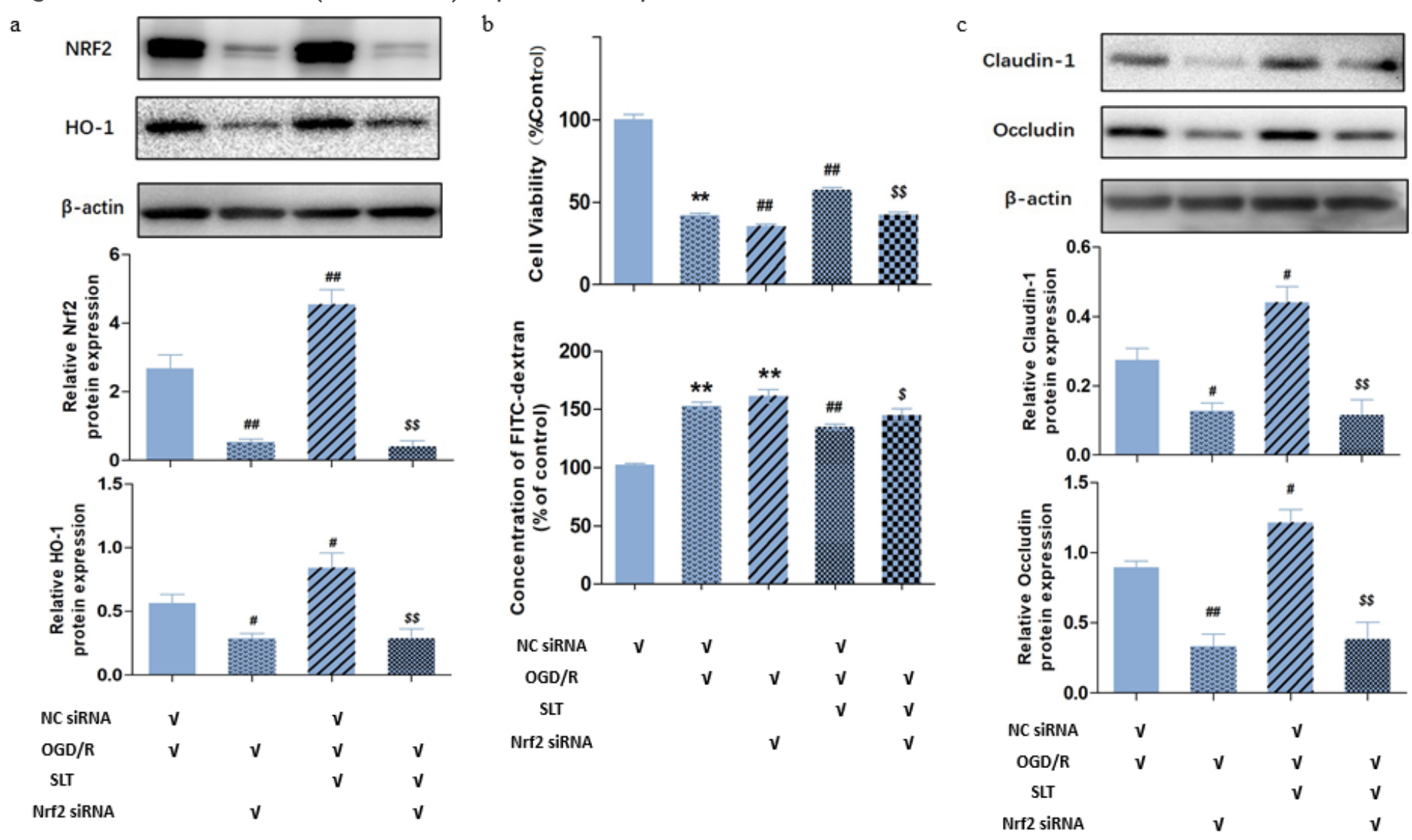

\section{Figure 6}

Blocking of Nrf2/HO-1 pathway ablated SLT's protection against OGD/R injury on cerebral microvascular endothelial cells. (a) Effects of Nrf2 siRNA interference on the increases of Nrf2 and HO-1 expressions by SLT's in OGD/R injured hCMEC/D3 cells $(n=3)$. (b) Effects Nrf2 siRNA interference on SLT's amelioration on cell viabilities and FITC-dextran permeabilities in OGD/R injured hCMEC/D3 cells $(n=6)$. (c) Effect of Nrf2 siRNA interference on SLT's amelioration on claudin-1 and occludin expressions in OGD/R injured hCMEC/D3 cells $(n=3)$. Data are expressed as mean $\pm S E M .{ }^{*} p<0.05,{ }^{*} p<0.01$ vs. negative control siRNA 
(NC siRNA) group; \#p<0.05, \#\#p<0.01 vs. NC siRNA+OGD/R group; $\$ p<0.05, \$ p<0.01$, vs. NC siRNA+OGD/R+SLT group.

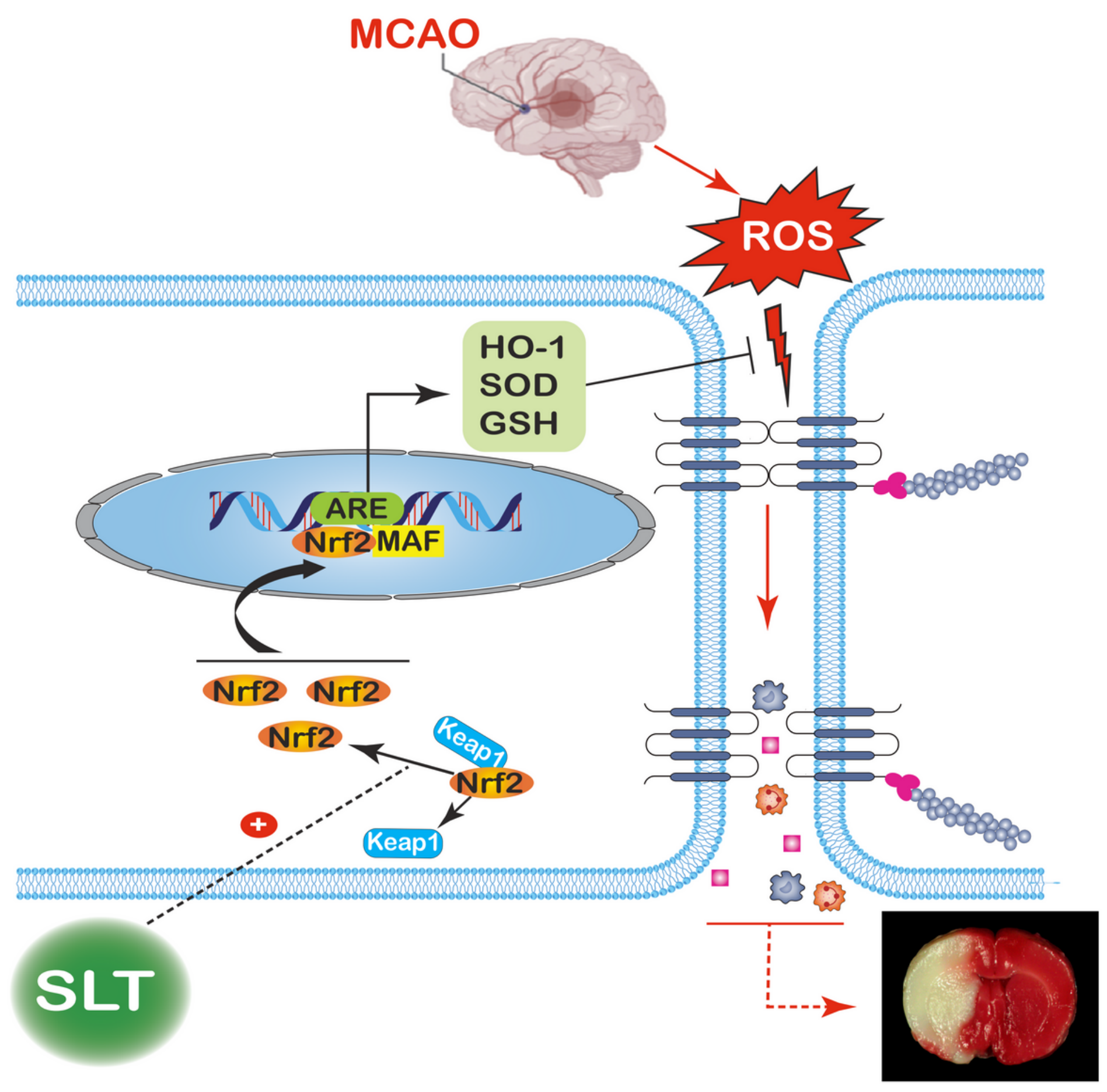

Figure 7

The putative mechanism for the therapeutic effect of SLT on brain ischemia. Occlusions in the cerebral arteries induce a drastic elevation in ROS contents; the excess ROS severely disrupts the brain blood barriers, leading to serious leakages from the blood vessels and brain edema, finally resulting in an infarction of large volume. SLT dramatically releases Nrf2 detained in the cytoplasm and promotes its nucleus translocation, thus priming the downstream transcriptions and increasing the contents of $\mathrm{HO}-1$, 
SOD, and GSH, which then massively eliminate ROS, cutting off the injury cascade and finally rescuing the brain from ischemia. 